\title{
On The Circular Altitude of Graphs
}

\author{
Saeed Shaebani \\ School of Mathematics and Computer Science \\ Damghan University \\ P.O. Box 36716-41167, Damghan, Iran \\ shaebani@du.ac.ir
}

\begin{abstract}
Peter Cameron introduced the concept of the circular altitude of graphs [2]; a parameter which was shown by Bamberg et al. 11 that provides a lower bound on the circular chromatic number. In this note, we investigate this parameter and show that the circular altitude of a graph is equal to the maximum of circular altitudes of its blocks. Also, we show that homomorphically equivalent graphs have the same circular altitudes. Finally, we prove that the circular altitude of the Cartesian product of two graphs is equal to the maximum of circular altitudes of its factors.
\end{abstract}

Keywords: Circular altitude, Monotonic cycle, Block, Homomorphism, Cartesian product.

\section{Mathematics Subject Classification: 05C38, 05C40, 05C60, 05C76}

\section{Introduction}

Unless otherwise stated, considered graphs in this paper are simple and also have nonempty and finite vertex sets. Also, the vertex set and the edge set of a graph $G$ are denoted by the symbols $V(G)$ and $E(G)$, respectively.

Let $G$ be a graph and $\mathcal{U}:\{1,2, \ldots,|V(G)|\} \rightarrow V(G)$ be a bijection. For simplicity, for each $i$ in $\{1,2, \ldots,|V(G)|\}$, we write $u_{i}$ instead of $\mathcal{U}(i)$. By a $\mathcal{U}$-monotonic cycle of length $m$, we mean a sequence $u_{i_{1}}, u_{i_{2}}, \ldots, u_{i_{m}}$ of $m$ pairwise distinct vertices of $G$ such that whenever $m>1$, the following three conditions hold simultaneously :

- $i_{1}<i_{2}<\cdots<i_{m}$

- For each $t$ with $1 \leq t \leq m-1$, two vertices $u_{i_{t}}$ and $u_{i_{t+1}}$ are adjacent;

- Two vertices $u_{i_{m}}$ and $u_{i_{1}}$ are adjacent.

One can consider the bijection $\mathcal{U}$ as a linear ordering of $V(G)$ like $u_{1}, u_{2}, \ldots, u_{|V(G)|}$; and it can be thought of a $\mathcal{U}$-monotonic cycle of length $m$ as a single vertex $u_{i_{1}}$ (for $m=1$ ), an edge $u_{i_{1}} u_{i_{2}}$ with $i_{1}<i_{2}$ (for $m=2$ ), or a cycle $u_{i_{1}} \sim u_{i_{2}} \sim$ $\cdots \sim u_{i_{m}} \sim u_{i_{1}}$ such that $u_{i_{1}}, u_{i_{2}}, \ldots, u_{i_{m}}$ appear according to the linear ordering $u_{1}, u_{2}, \ldots, u_{|V(G)|}($ for $m \geq 3$ ). For a linear ordering $\mathcal{U}:\{1,2, \ldots,|V(G)|\} \rightarrow V(G)$, the symbol $|\mathcal{U}|$ stands for the maximum length of a $\mathcal{U}$-monotonic cycle. The circular altitude of a graph $G$, denoted by $\alpha^{o}(G)$, is defined as the minimum of $|\mathcal{U}|$, where $\mathcal{U}$ ranges over all linear orderings of $V(G)$. In other words, the circular altitude of 
a graph $G$ is the maximum positive integer $m$ for which every linear ordering $\mathcal{U}$ of $V(G)$ admits a $\mathcal{U}$-monotonic cycle of length at least $m$.

Let $\mathcal{U}_{1}$ be a linear ordering of $V(G)$ of the form $u_{1}, u_{2}, \ldots, u_{|V(G)|}$. One can correspond this linear ordering to a circle clock of radius 1 for which $u_{1}, u_{2}, \ldots, u_{|V(G)|}$ are arranged clockwise around it with the additional property that $u_{1}$ is at the highest point of this circle clock. Now, by rotating all the arranged vertices counterclockwise $\frac{2 \pi}{k}$ radians, we obtain the linear ordering $\mathcal{U}_{2}$ of $V(G)$ of the form $u_{2}, u_{3}, \ldots, u_{|V(G)|}, u_{1}$. Continuing in this procedure $k-1$ times, we gain $\mathcal{U}_{k}$ as the linear ordering of $V(G)$ of the form $u_{k}, u_{k+1}, \ldots, u_{|V(G)|}, u_{1}, u_{2}, \ldots, u_{k-1}$. It is obvious that if there exists a $\mathcal{U}_{1}$-monotonic cycle of length $m$, then for each $k$ in $\{1,2, \ldots,|V(G)|\}$, there exist $\mathcal{U}_{k}$-monotonic cycles of length $m$, as well. Therefore, $\left|\mathcal{U}_{1}\right|=\left|\mathcal{U}_{2}\right|=\cdots=\left|\mathcal{U}_{|V(G)|}\right|$. This fact shows that for determining the circular altitude of $G$, if we regard an arbitrary vertex $x$ in $V(G)$ as fixed, we may just restrict our attention to those linear orderings whose first terms are $x$; i.e., those linear orderings that begin with $x$. Also, $\left|\mathcal{U}_{1}\right|=\left|\mathcal{U}_{2}\right|=\cdots=\left|\mathcal{U}_{|V(G)|}\right|$ implies that one may just focus on circular orderings of $V(G)$ instead of linear orderings of $V(G)$, and then consider monotonic cycles in these circular orderings. Nevertheless, for the sake of simplification, we just use linear orderings rather than circular orderings.

An interesting property of the citcular altitude parameter is its monotonicity. This parameter is increasing in the sense that if a graph $H$ is a subgraph of a graph $G$, then $\alpha^{o}(H) \leq \alpha^{o}(G)$. As a consequence, the clique number is a lower bound for the circular altitude. So, the inequality $\omega(G) \leq \alpha^{o}(G)$ holds for every graph $G$. Also, since the circular altitude is a parameter related to monotonic cycles, it is a natural proposition that if $\alpha^{o}(G) \geq 3$, then $\alpha^{o}(G)$ is greater than or equal to the girth of $G[1]$.

The concept of circular altitude of graphs was introduced by Cameron in [2] and it was investigated by Bamberg, Corr, Devillers, Hawtin, Pivotto, and Swartz in [1]. Bamberg et al. showed that the circular altitude of a graph provides a lower bound on its circular chromatic number; that is, $\alpha^{o}(G) \leq \chi_{c}(G)$. Also, they provided some examples of graphs with $\omega(G)<\alpha^{o}(G)<\chi_{c}(G)$.

In this paper, we investigate the circular altitude of graphs. We prove that the circular altitude of a graph is equal to the maximum of circular altitudes of its blocks. Also, we show that homomorphically equivalent graphs have the same circular altitudes. Finally, we prove that the circular altitude of the Cartesian product of two graphs is equal to the maximum of circular altitudes of its factors.

\section{Reduction to 2-connected graphs}

This section concerns the relation between the circular altitude of a graph and circular altitudes of its blocks. We show that for the circular altitude parameter, we can restrict the study of all graphs just to the study of their blocks.

A cut-vertex of a graph, is a vertex in such a way that removing it from the graph results in a new graph with a higher number of components. A graph is called nonseparable if it is connected and has no cut-vertices. A block of a graph $G$ is a nonseparable subgraph of $G$ which is not a subgraph of another nonseparable subgraph of $G$. In other words, a block of a graph $G$ is a maximal nonseparable 
subgraph of $G$. Every block of a graph is a complete graph with one vertex or is a complete graph with two vertices or is a 2-connected graph.

In the next theorem, we show that the circular altitude of every graph equals the maximum of circular altitudes of its blocks. Since $\alpha^{o}\left(K_{1}\right)=1$ and $\alpha^{o}\left(K_{2}\right)=2$, therefore, the study of circular altitude of graphs can be reduced to the study of circular altitude of 2-connected graphs.

Theorem 1. The circular altitude of every graph equals the maximum of circular altitudes of its blocks.

Proof. The proof falls into three independent steps, as follows.

Step 1. In this step, we show that the circular altitude of a graph $G$ is equal to the maximum of circular altitudes of its components. In this regard, suppose that all components of $G$ are $G_{1}, G_{2}, \ldots, G_{m}$. Since $G_{1}, G_{2}, \ldots, G_{m}$ are subgraphs of $G$, we have

$$
\alpha^{o}(G) \geq \max \left\{\alpha^{o}\left(G_{1}\right), \alpha^{o}\left(G_{2}\right), \ldots, \alpha^{o}\left(G_{m}\right)\right\} .
$$

What is left in this step is to show that $\alpha^{o}(G) \leq \max \left\{\alpha^{o}\left(G_{1}\right), \alpha^{o}\left(G_{2}\right), \ldots, \alpha^{o}\left(G_{m}\right)\right\}$. For each $i$ in $\{1, \ldots, m\}$ let the bijection $\mathcal{U}_{i}:\left\{1,2, \ldots,\left|V\left(G_{i}\right)\right|\right\} \rightarrow V\left(G_{i}\right)$, which is considered as $v_{i_{1}}, v_{i_{2}}, \ldots, v_{i_{\mid V\left(G_{i}\right)}}$, be a linear ordering of $V\left(G_{i}\right)$ in such a way that $\alpha^{o}\left(G_{i}\right)=\left|\mathcal{U}_{i}\right|$. Therefore, for each $i$ in $\{1, \ldots, m\}$, the maximum length of a $\mathcal{U}_{i}$-monotonic cycle equals $\alpha^{o}\left(G_{i}\right)$. Now, according to the linear orderings $\mathcal{U}_{1}, \mathcal{U}_{2}, \ldots, \mathcal{U}_{m}$, regard the natural linear ordering $\mathcal{U}:=\mathcal{U}_{1} \mathcal{U}_{2} \ldots \mathcal{U}_{m}$ as a linear ordering of $V(G)$. The linear ordering $\mathcal{U}$ satisfies both of the following two conditions :

- If $1 \leq i<j \leq m$ then each vertex of $G_{i}$ occurs before each vertex of $G_{j}$.

- For each $i$ in $\{1, \ldots, m\}$ and for any two vertices $a$ and $b$ in $V\left(G_{i}\right)$, the vertex $a$ occurs before the vertex $b$ in the linear ordering $\mathcal{U}$ iff $a$ occurs before $b$ in the linear ordering $\mathcal{U}_{i}$.

Since each $\mathcal{U}$-monotonic cycle lies in exactly one of $G_{1}, G_{2}, \ldots, G_{m}$, we obtain

$$
|\mathcal{U}| \leq \max \left\{\left|\mathcal{U}_{1}\right|,\left|\mathcal{U}_{2}\right|, \ldots,\left|\mathcal{U}_{m}\right|\right\} .
$$

Therefore,

$$
\alpha^{o}(G) \leq|\mathcal{U}| \leq \max \left\{\alpha^{o}\left(G_{1}\right), \alpha^{o}\left(G_{2}\right), \ldots, \alpha^{o}\left(G_{m}\right)\right\} .
$$

So, $\alpha^{o}(G)=\max \left\{\alpha^{o}\left(G_{1}\right), \alpha^{o}\left(G_{2}\right), \ldots, \alpha^{o}\left(G_{m}\right)\right\}$; which is desired in this step.

Step 2. In this step, we suppose that $H$ is a connected graph with a cut-vertex $x$, and $H_{1}, H_{2}, \ldots, H_{m}$ are all components of $H-\{x\}$, where $H-\{x\}$ is the graph obtained by deleting the vertex $x$ from the graph $H$. Also, for each $i$ in $\{1, \ldots, m\}$, let $H_{i}^{\prime}$ be the subgraph of $H$ induced by $V\left(H_{i}\right) \cup\{x\}$. In this step, the aim is proving that $\alpha^{o}(H)=\max \left\{\alpha^{o}\left(H_{1}^{\prime}\right), \alpha^{o}\left(H_{2}^{\prime}\right), \ldots, \alpha^{o}\left(H_{m}^{\prime}\right)\right\}$. For proving this fact, we should mention that for each of the graphs $H, H_{1}^{\prime}, H_{2}^{\prime}, \ldots, H_{m}^{\prime}$, without loss of generality, we can just restrict our attention to those linear orderings that begin with $x$. For each $i$ in $\{1,2, \ldots, m\}$, let $\mathcal{W}_{i}$ be a linear ordering of $V\left(H_{i}^{\prime}\right)$ that begins with $x$ and also $\alpha^{o}\left(H_{i}^{\prime}\right)=\left|\mathcal{W}_{i}\right|$. Now, using $\mathcal{W}_{1}, \mathcal{W}_{2}, \ldots, \mathcal{W}_{m}$, we consider a linear ordering $\mathcal{W}$ of $V(H)$ that satisfies the following two conditions simultaneously: 
- The linear ordering $\mathcal{W}$ begins with $x$.

- For each $i$ in $\{1,2, \ldots, m\}$ and for any two vertices $a$ and $b$ in $V\left(H_{i}^{\prime}\right)$, the vertex $a$ occurs before the vertex $b$ in the linear ordering $\mathcal{W}$ iff $a$ occurs before $b$ in the linear ordering $\mathcal{W}_{i}$.

Now, since any $\mathcal{W}$-monotonic cycle whose length is $|\mathcal{W}|$ settles in exactly one of $H_{1}^{\prime}, H_{2}^{\prime}, \ldots, H_{m}^{\prime}$, the inequality $|\mathcal{W}| \leq \max \left\{\left|\mathcal{W}_{1}\right|,\left|\mathcal{W}_{2}\right|, \ldots,\left|\mathcal{W}_{m}\right|\right\}$ holds. Thus,

$$
\alpha^{o}(H) \leq|\mathcal{W}| \leq \max \left\{\alpha^{o}\left(H_{1}^{\prime}\right), \alpha^{o}\left(H_{2}^{\prime}\right), \ldots, \alpha^{o}\left(H_{m}^{\prime}\right)\right\} .
$$

On the other hand, since $H_{1}^{\prime}, H_{2}^{\prime}, \ldots, H_{m}^{\prime}$ are subgraphs of $H$, we have

$$
\alpha^{o}(H) \geq \max \left\{\alpha^{o}\left(H_{1}^{\prime}\right), \alpha^{o}\left(H_{2}^{\prime}\right), \ldots, \alpha^{o}\left(H_{m}^{\prime}\right)\right\} .
$$

Hence, $\alpha^{o}(H)=\max \left\{\alpha^{o}\left(H_{1}^{\prime}\right), \alpha^{o}\left(H_{2}^{\prime}\right), \ldots, \alpha^{o}\left(H_{m}^{\prime}\right)\right\}$; and we are done in this step.

We are now in a position to complete the proof of the theorem in Step 3.

Step 3. Let $\mathcal{G}$ be a graph. We show that $\alpha^{o}(\mathcal{G})=\max \left\{\alpha^{o}(\mathcal{B}) \mid \mathcal{B}\right.$ is a block of $\left.\mathcal{G}\right\}$. The inequality $\alpha^{o}(\mathcal{G}) \geq \max \left\{\alpha^{o}(\mathcal{B}) \mid \mathcal{B}\right.$ is a block of $\left.\mathcal{G}\right\}$ follows immediately from the fact that each block of $\mathcal{G}$ is a subgraph of $\mathcal{G}$.

For proving $\alpha^{o}(\mathcal{G})=\max \left\{\alpha^{o}(\mathcal{B}) \mid \mathcal{B}\right.$ is a block of $\left.\mathcal{G}\right\}$, it is sufficient to show that there exists a block of $\mathcal{G}$ whose circular altitude equals $\alpha^{o}(\mathcal{G})$. In this regard, define $\mathcal{S}$ as the set of all subgraphs of $\mathcal{G}$ such that their circular altitudes are equal to $\alpha^{o}(\mathcal{G})$. More precisely,

$$
\mathcal{S}:=\left\{\mathcal{T} \mid \mathcal{T} \text { is a subgraph of } \mathcal{G} \text { and } \alpha^{o}(\mathcal{T})=\alpha^{o}(\mathcal{G})\right\}
$$

Let us regard a graph $\mathcal{H}$ in $\mathcal{S}$ with the minimum number of vertices. Indeed, $\mathcal{H}$ is a subgraph of $\mathcal{G}$ with the minimum number of vertices for which $\alpha^{o}(\mathcal{H})=\alpha^{o}(\mathcal{G})$. According to the Step 1, the graph $\mathcal{H}$ is connected; since otherwise, there exists a component of $\mathcal{H}$ (with a smaller number of vertices than to $|V(\mathcal{H})|$ ) such that its circular altitude equals $\alpha^{o}(\mathcal{G})$, which is a contradiction to the minimality of the number of vertices of $\mathcal{H}$. Also, on account of the Step 2, the graph $\mathcal{H}$ has not any cutvertices. So, $\mathcal{H}$ is a subgraph of $\mathcal{G}$ which is connected and has not any cut-vertices. Consequently, $\mathcal{H}$ is a subgraph of a block $\mathcal{B}$ of $\mathcal{G}$. Now, since $\mathcal{H}$ is a subgraph of $\mathcal{B}$ and $\mathcal{B}$ is a subgraph of $\mathcal{G}$, we conclude that $\alpha^{o}(\mathcal{G})=\alpha^{o}(\mathcal{H}) \leq \alpha^{o}(\mathcal{B}) \leq \alpha^{o}(\mathcal{G})$. Accordingly, $\alpha^{o}(\mathcal{B})=\alpha^{o}(\mathcal{G})$; and the assertion follows.

\section{Circular altitude and graph homomorphism}

A homomorphism from a graph $G$ to a graph $H$, is a function from the vertex set of $G$ to the vertex set of $H$ which preserves all adjacencies. To be more precise, a homomorphism from a graph $G$ to a graph $H$ is a function $f: V(G) \rightarrow V(H)$ such that for any two vertices $x$ and $y$ in $V(G)$ the following condition holds

$$
x y \in E(G) \Longrightarrow f(x) f(y) \in E(H) .
$$

We write $G \longrightarrow H$ whenever there exists a graph homomorphism from $G$ to $H$. Two graphs $G$ and $H$ are called homomorphically equivalent graphs if both of the conditions $G \longrightarrow H$ and $H \longrightarrow G$ are satisfied. The theory of graph homomorphisms 
is one of the most interesting concepts in graph theory. Many important notions and parameters in graph theory can be interpreted in terms of graph homomorphisms. For various examples, one can see the references [3, 4, 6]. For the circular altitude parameter, a natural question is whether homomorphically equivalent graphs have the same circular altitudes. The next theorem gives an affirmative answer to this interesting question.

Theorem 2. Homomorphically equivalent graphs have the same circular altitudes.

Proof. In order to prove the theorem, it is sufficient to show that if there exists a surjective homomorphism $f: V(G) \rightarrow V(H)$ from a graph $G$ to a graph $H$, then $\alpha^{o}(G) \leq \alpha^{o}(H)$. In this regard, let $\mathcal{U}$ be a linear ordering of $V(H)$ with $|\mathcal{U}|=\alpha^{o}(H)$. Consider the linear ordering $\mathcal{U}$ as $v_{1}, v_{2}, \ldots, v_{|V(H)|}$. Now, making use of the linear ordering $\mathcal{U}$, we construct a linear ordering $\widetilde{\mathcal{U}}$ of $V(G)$ as $f^{-1}\left(v_{1}\right) f^{-1}\left(v_{2}\right) \cdots f^{-1}\left(v_{|V(H)|}\right)$. In the linear ordering $\widetilde{\mathcal{U}}$, for each $i$ and $j$ with $1 \leq i<j \leq|V(H)|$, each vertex of $f^{-1}\left(v_{i}\right)$ occurs before each vertex of $f^{-1}\left(v_{j}\right)$. Indeed, first all vertices of $f^{-1}\left(v_{1}\right)$ are arbitrarily arranged, then all vertices of $f^{-1}\left(v_{2}\right)$ are arbitrarily arranged, ... , and finally all vertices of $f^{-1}\left(v_{|V(H)|}\right)$ are arbitrarily arranged in order to construct $\widetilde{\mathcal{U}}$. The homomorphism $f: V(G) \rightarrow V(H)$ is a function from $V(G)$ onto $V(H)$ and the restriction of $f$ on the vertices of any $\widetilde{\mathcal{U}}$ monotonic cycle is an injective function. Furthermore, by the homomorphism $f$, the image of any $\widetilde{\mathcal{U}}$-monotonic cycle of length $m$ forms a $\mathcal{U}$-monotonic cycle of length $m$. Accordingly, $|\widetilde{\mathcal{U}}| \leq|\mathcal{U}|$. Since $|\mathcal{U}|=\alpha^{o}(H)$, we conclude that $\alpha^{o}(G) \leq|\widetilde{\mathcal{U}}| \leq \alpha^{o}(H)$; and the assertion follows.

In view of the proof of the Theorem 2, we observe that the circular altitude of graphs is an increasing parameter in the sense that if there exists a homomorphism from a graph $G$ to a graph $H$, then $\alpha^{o}(G) \leq \alpha^{o}(H)$. Indeed,

$$
G \longrightarrow H \quad \alpha^{o}(G) \leq \alpha^{o}(H) .
$$

Theorem 1 states that the circular altitude of every graph equals the maximum of circular altitudes of its blocks. A natural question is the relation between the circular altitude of a graph and the circular altitudes of its cores. A graph $G$ is called a core graph whenever every homomorphism from $G$ to itself is an isomorphism. A core of a graph $\mathcal{G}$, is a subgraph of $\mathcal{G}$, say $G$, such that the following two conditions hold simultaneously :

- $G$ is a core graph,

- $G$ and $\mathcal{G}$ are homomorphically equivalent.

In other words, a core of a graph $\mathcal{G}$ is a subgraph $G$ of $\mathcal{G}$ such that $G$ is a core graph and $\mathcal{G} \longrightarrow G$. It is known that every graph has a unique core up to isomorphism; see [3] or [4] or [6]. So, Theorem 2 implies that the circular altitude of any graph is equal to the circular altitude of its core.

Corollary 1. The circular altitude of any graph is equal to the circular altitude of its core. 


\section{Circular altitude of Cartesian product of graphs}

Let $G$ and $H$ be two graphs. The Cartesian product of $G$ and $H$, denoted by $G \square H$, is a graph whose vertex set is $V(G) \times V(H)$, and two vertices $\left(v_{1}, h_{1}\right)$ and $\left(v_{2}, h_{2}\right)$ are adjacent in $G \square H$ if one of the following two conditions holds :

- $v_{1}=v_{2}$ and $h_{1} h_{2} \in E(H)$,

- $h_{1}=h_{2}$ and $v_{1} v_{2} \in E(G)$.

The graphs $G$ and $H$ are called the factors of the Cartesian product $G \square H$. The Cartesian product of graphs is commutative up to isomorphism; that is, $G \square H$ and $H \square G$ are isomorphic for any two graphs $G$ and $H$. Also, it is associative up to isomorphism. For more information about Cartesian product of graphs, one can see [5].

In 1957, Sabidussi proved that the chromatic number of the Cartesian product of two graphs is equal to the maximum of chromatic numbers of its factors [7]. More Precisely, for any two graphs $G$ and $H$, we have $\chi(G \square H)=\max \{\chi(G), \chi(H)\}$. Zhu showed that this fact also holds for the circular chromatic number; that is, for any two graphs $G$ and $H$, the equality $\chi_{c}(G \square H)=\max \left\{\chi_{c}(G), \chi_{c}(H)\right\}$ holds [8]. In the next theorem, we are concerned with how circular altitude behaves on Cartesian product of graphs. We prove an analogue of the two mentioned equalities for the circular altitude parameter.

Theorem 3. The circular altitude of the Cartesian product of two graphs is equal to the maximum of circular altitudes of its factors. Indeed, for any two graphs $G$ and $H$ we have $\alpha^{o}(G \square H)=\max \left\{\alpha^{o}(G), \alpha^{o}(H)\right\}$.

Proof. Let the vertex sets of graphs $G$ and $H$ be $V(G):=\left\{g_{1}, g_{2}, \ldots, g_{|V(G)|}\right\}$ and $V(H):=\left\{h_{1}, h_{2}, \ldots, h_{|V(H)|}\right\}$, respectively. For each integer $i$ in $\{1,2, \ldots,|V(G)|\}$, the subgraph of $G \square H$ induced by the set $\left\{g_{i}\right\} \times V(H)$ is isomorphic to $H$. Denote this subgraph by $\mathcal{H}_{i}$. This shows that $\alpha^{o}(H) \leq \alpha^{o}(G \square H)$. Also, for each $j$ in $\{1,2, \ldots,|V(H)|\}$, the subgraph of $G \square H$ induced by the set $V(G) \times\left\{h_{j}\right\}$ is isomorphic to $G$. Similarly, we denote this subgraph by $\mathcal{G}_{j}$. Thus, $\alpha^{o}(G) \leq \alpha^{o}(G \square H)$. We deduce that

$$
\alpha^{o}(G \square H) \geq \max \left\{\alpha^{o}(G), \alpha^{o}(H)\right\} .
$$

So, we shall have established the theorem if we prove the following :

$$
\alpha^{o}(G \square H) \leq \max \left\{\alpha^{o}(G), \alpha^{o}(H)\right\} .
$$

In this regard, we consider below three cases.

Case 1. The case that $\alpha^{o}(G)=1$.

In this case, $G$ has no edges and each component of $G \square H$ is isomorphic to the graph $H$. In view of the proof of the Theorem 1, we find that the circular altitude of every graph equals the maximum of circular altitudes of its components. This shows that in this case, for the graph $G \square H$ we have

$$
\alpha^{o}(G \square H)=\alpha^{o}(H) \leq \max \left\{\alpha^{o}(G), \alpha^{o}(H)\right\} .
$$


Case 2. The case that $\alpha^{o}(H)=1$.

Since $G \square H$ and $H \square G$ are isomorphic, similar to the previous case,

$$
\alpha^{o}(G \square H)=\alpha^{o}(H \square G)=\alpha^{o}(G) \leq \max \left\{\alpha^{o}(G), \alpha^{o}(H)\right\} .
$$

Case 3. The case that $\alpha^{o}(G) \geq 2$ and $\alpha^{o}(H) \geq 2$.

Without loss of generality, we may assume that $\mathcal{U}_{G}:=g_{1}, g_{2}, \ldots, g_{|V(G)|}$ and $\mathcal{U}_{H}:=$ $h_{1}, h_{2}, \ldots, h_{|V(H)|}$ are linear orderings of $G$ and $H$, respectively, in such a way that $\alpha^{o}(G)=\left|\mathcal{U}_{G}\right|$ and $\alpha^{o}(H)=\left|\mathcal{U}_{H}\right|$. Let us consider the linear ordering $\mathcal{U}_{G \square H}$ of $V(G \square H)$ for which the vertices of $V(G \square H)$ are ordered lexicographically. More precisely, in the linear ordering $\mathcal{U}_{G \square H}$, a vertex $\left(g_{i}, h_{j}\right)$ occurs before a vertex $\left(g_{i^{\prime}}, h_{j^{\prime}}\right)$ iff one of the following two conditions holds :

- $i<i^{\prime}$

- $i=i^{\prime}$ and $j<j^{\prime}$.

Certainly, the inequality $\alpha^{o}(G \square H) \leq\left|\mathcal{U}_{G \square H}\right|$ holds. Our aim is showing that $\left|\mathcal{U}_{G \square H}\right| \leq \max \left\{\alpha^{o}(G), \alpha^{o}(H)\right\}$. The procedure is regarding an arbitrary $\mathcal{U}_{G \square H^{-}}$ monotonic cycle $\left(g_{i_{1}}, h_{j_{1}}\right),\left(g_{i_{2}}, h_{j_{2}}\right), \ldots,\left(g_{i_{m}}, h_{j_{m}}\right)$ as fixed and proving that $m \leq$ $\max \left\{\alpha^{o}(G), \alpha^{o}(H)\right\}$.

There is nothing to prove when $m=1$. So, assume that $m \geq 2$. The vertices of $V(G \square H)$ are ordered lexicographically in the linear ordering $\mathcal{U}_{G \square H}$. Hence, $i_{1} \leq$ $i_{2} \leq \cdots \leq i_{m}$. We show that also $j_{1} \leq j_{2} \leq \cdots \leq j_{m}$ holds as well. Let $k$ be an integer in $\{1,2, \ldots, m-1\}$. Obviously, since $\left(g_{i_{k}}, h_{j_{k}}\right)$ and $\left(g_{i_{k+1}}, h_{j_{k+1}}\right)$ are adjacent in $G \square H$, we have either $h_{j_{k}}=h_{j_{k+1}}$ or $g_{i_{k}}=g_{i_{k+1}}$. The former implies $j_{k}=j_{k+1}$. The latter imples $i_{k}=i_{k+1}$; and since $\left(g_{i_{k}}, h_{j_{k}}\right)$ occurs before $\left(g_{i_{k+1}}, h_{j_{k+1}}\right)$, we must have $j_{k}<j_{k+1}$. We conclude that $j_{1} \leq j_{2} \leq \cdots \leq j_{m}$.

Now, since the sequence $\left(g_{i_{1}}, h_{j_{1}}\right),\left(g_{i_{2}}, h_{j_{2}}\right), \ldots,\left(g_{i_{m}}, h_{j_{m}}\right)$ is a $\mathcal{U}_{G \square H}$-monotonic cycle, two vertices $\left(g_{i_{1}}, h_{j_{1}}\right)$ and $\left(g_{i_{m}}, h_{j_{m}}\right)$ are adjacent in $G \square H$. Therefore, one of the following two subcases holds :

Subcase 3.1. Two vertices $h_{j_{1}}$ and $h_{j_{m}}$ are adjacent in $H$, and $g_{i_{1}}=g_{i_{m}}$. In this subcase, since $g_{i_{1}}=g_{i_{m}}$, we have $i_{1}=i_{m}$; and therefore, $i_{1}=i_{2}=$ $\cdots=i_{m}$. So, $g_{i_{1}}=g_{i_{2}}=\cdots=g_{i_{m}}$; and thus, the $\mathcal{U}_{G \square H^{-m o n o t o n i c} \text { cycle }}$ $\left(g_{i_{1}}, h_{j_{1}}\right),\left(g_{i_{2}}, h_{j_{2}}\right), \ldots,\left(g_{i_{m}}, h_{j_{m}}\right)$ lies in $\mathcal{H}_{i_{1}}$. Besides, the sequence $h_{j_{1}}, h_{j_{2}}, \ldots, h_{j_{m}}$ forms a $\mathcal{U}_{H}$-monotonic cycle. Therefore,

$$
m \leq\left|\mathcal{U}_{H}\right|=\alpha^{o}(H) \leq \max \left\{\alpha^{o}(G), \alpha^{o}(H)\right\} .
$$

Subcase 3.2. Two vertices $g_{i_{1}}$ and $g_{i_{m}}$ are adjacent in $G$, and $h_{j_{1}}=h_{j_{m}}$.

In this subcase, the equality $h_{j_{1}}=h_{j_{m}}$ implies that $j_{1}=j_{m}$. So, $j_{1}=j_{2}=$ $\cdots=j_{m}$ and $h_{j_{1}}=h_{j_{2}}=\cdots=h_{j_{m}}$. This shows that the $\mathcal{U}_{G \square H^{-m o n o t o n i c ~ c y c l e ~}}$ $\left(g_{i_{1}}, h_{j_{1}}\right),\left(g_{i_{2}}, h_{j_{2}}\right), \ldots,\left(g_{i_{m}}, h_{j_{m}}\right)$ lies in $\mathcal{G}_{j_{1}}$; and also, the sequence $g_{i_{1}}, g_{i_{2}}, \ldots, g_{i_{m}}$ forms a $\mathcal{U}_{G}$-monotonic cycle. Accordingly,

$$
m \leq\left|\mathcal{U}_{G}\right|=\alpha^{o}(G) \leq \max \left\{\alpha^{o}(G), \alpha^{o}(H)\right\} ;
$$

which completes the proof of the theorem. 


\section{References}

[1] J. Bamberg, B. Corr, A. Devillers, D. Hawtin, I. Pivotto, and E. Swartz, The circular altitude of a graph, arXiv:1608.06127v1 (2016).

[2] P. J. Cameron, Altitude and chromatic number, Available at http://www.maths.qmul.ac.uk/ pjc/preprints/valt.pdf (May 2004).

[3] C. Godsil and G. Royle, Algebraic graph theory, Springer-Verlag, New York (2001).

[4] G. Hahn and C. Tardif, Graph homomorphisms: structure and symmetry, in Graph Symmetry, G. Hahn and G. Sabidussi, eds., no. 497 in NATO Adv. Sci. Inst. Ser. C Math. Phys. Sci., Kluwer, Dordrecht (1997), 107-167.

[5] R. Hammack, W. Imrich, and S. Klavžar, Handbook of product graphs, Discrete Mathematics and its Applications (Boca Raton). CRC Press, Boca Raton, FL, second edition (2011).

[6] P. Hell and J. Nešetřil, Graphs and homomorphisms, Oxford Lecture Series in Mathematics and its Applications 28, Oxford University Press, Oxford (2004).

[7] G. Sabidussi, Graphs with given group and given graph-theoretical properties, Canad. J. Math. 9 (1957), 515-525.

[8] X. Zhu, Circular chromatic number: a survey, Discrete Mathematics 229 (2001), 371-410. 